\title{
A Call to Action: The Role of Healthcare Providers in Reducing the Burden Associated with Neurogenic Orthostatic Hypotension
}

\author{
Jessie S. Gibson (D) - Christine B. Hunter - L. Arthur Hewitt
}

Received: April 30, 2020 / Published online: July 27, 2020

(c) The Author(s) 2020 better quality of life for patients. To better understand the manifestations and real-life impact of living with nOH symptoms, perspectives from a patient with $\mathrm{nOH}$ and his caregiver (wife) are provided, along with key findings from a published survey of patients and caregivers on the burden of $\mathrm{nOH}$. In addition, insights and advice on a practical approach for diagnosing, educating, and treating patients with $\mathrm{nOH}$ are outlined.

\section{PLAIN LANGUAGE SUMMARY}

This article discusses what neurogenic orthostatic hypotension $(\mathrm{nOH})$ is, its symptoms, and how patients and healthcare providers (HCPs) can work together to manage $\mathrm{nOH}$ symptoms. What is nOH? People with neurologic disorders, like Parkinson disease, may also have a separate condition called nOH. nOH occurs when blood pressure drops too much when standing up after sitting or lying down. What are the symptoms of $\mathrm{nOH}$, and how can they affect people's lives? Most often, people with nOH report feeling lightheaded, faint, or dizzy when standing up. Other symptoms of $\mathrm{nOH}$ include pain in the shoulders and neck, trouble thinking clearly, tiredness, or blurry vision. The symptoms of $\mathrm{nOH}$ can cause falls and injuries. Because of nOH symptoms, people may be 
unable to do daily activities and may feel worried or anxious. What do patients and HCPs need to know when discussing nOH symptoms? A survey showed that patients may not talk about their nOH symptoms with their HCPs unless symptoms are severe, and that patients can find receiving a diagnosis of $\mathrm{nOH}$ challenging. Patients can track nOH symptoms in a daily journal to use in discussions with their HCPs. How can HCPs help patients with nOH? HCPs can ask patients with underlying neurologic disorders about symptoms that occur on standing and confirm a diagnosis of $\mathrm{nOH}$ by comparing the patient's blood pressures measured lying down and after standing up. HCPs can provide information about lifestyle changes and medications that are available to manage $\mathrm{nOH}$ symptoms.

Keywords: Caregiver; Healthcare providers; Neurogenic orthostatic hypotension; Parkinson disease; Patient perspective; Quality of life; Symptom burden

\section{Key Summary Points}

Neurogenic orthostatic hypotension (nOH) commonly occurs in patients with neurodegenerative diseases (e.g., Parkinson disease) as a consequence of autonomic nervous system dysfunction.

Patients with $\mathrm{nOH}$ may experience severe and burdensome symptoms, which can result in functional impairment and decreased quality of life.

Patients may not recognize $\mathrm{nOH}$ and instead attribute their symptoms to the underlying neurologic condition (e.g., Parkinson disease).

By increasing healthcare providers' awareness about recognizing, diagnosing, and managing $\mathrm{nOH}$, clinical outcomes and quality of life in the patients they treat may be improved.
Treatment of nOH symptoms using nonpharmacologic and pharmacologic options may help improve patients' ability to perform daily activities and increase their sense of well-being.

\section{INTRODUCTION}

Aging is associated with an increased likelihood of synucleinopathies (e.g., Parkinson disease [PD]) [1], which can result in autonomic dysfunction [2]. A common consequence of this autonomic dysfunction is neurogenic orthostatic hypotension $(\mathrm{nOH})$, a condition in which orthostatic blood pressure (BP) drops [3]. Healthcare providers (HCPs) may frequently encounter patients with $\mathrm{nOH}$, but the condition may be under-recognized and, consequently, undertreated in clinical practice, despite its severe functional and psychosocial effects on patients [4]. HCPs caring for patients with PD and other neurodegenerative conditions can play a fundamental role in the recognition of $\mathrm{nOH}$ because of their frequent and in-depth interactions with these patients and their caregivers. However, patients may be unaware of or reluctant to discuss their $\mathrm{nOH}$ symptoms with their HCPs. Greater understanding of these communication barriers and ways to overcome these challenges, especially among HCPs practicing in neurology settings, may lead to more timely diagnosis of $\mathrm{nOH}$ and improved patient outcomes.

\section{SYMPTOMS OF nOH AND THEIR EFFECT}

The cardinal symptoms of $\mathrm{nOH}$ are dizziness and lightheadedness; however, the BP reduction and resulting organ hypoperfusion associated with $\mathrm{nOH}$ can cause a variety of nonspecific symptoms, including cognitive difficulties, neck and shoulder pain, fatigue, and visual disturbances [2]. The burden of $\mathrm{nOH}$ was examined in a survey of 363 patients with an underlying 
autonomic failure diagnosis and a formal diagnosis of nOH/orthostatic hypotension $(\mathrm{OH})$ or characteristic signs and/or symptoms of nOH/ $\mathrm{OH}$ (e.g., low BP, dizziness/lightheadedness), and 128 caregivers of such patients. In this survey, between 20 and $40 \%$ of patients reported persistently experiencing these types of symptoms (i.e., multiple times a day when moving to a sitting or standing position) [4]. $\mathrm{nOH}$ symptoms can increase the risk for falls and related injuries, a particular concern in older persons [2]. In the above-mentioned $\mathrm{nOH}$ burden survey, nearly $60 \%$ of patients and $80 \%$ of caregivers reported $\geq 1$ patient fall in the previous year [4]. Although falls due to $\mathrm{nOH}$ symptoms can have direct physical, financial, and psychosocial consequences (e.g., disability, mortality, increased healthcare costs, fear of falling, and subsequent limitation of activities) $[2,5-7]$, this survey also provided insight into the overall patient burden of nOH. Most patient and caregiver respondents $(>87 \%)$ reported that nOH symptoms impeded the patient's ability to perform everyday activities (e.g., physical activity/exercise, housework, traveling, leaving the house) [4]. Further, substantial percentages of patients and caregivers indicated that nOH symptoms affected patients by diminishing quality of life, causing a perceived loss of independence, and changing their lives drastically $(40-75 \%)$. Half of patients reported feeling anxious, worried, depressed, or discouraged because of nOH symptoms [4]. The experience of living with $\mathrm{nOH}$ symptoms is illustrated in the following patient perspective.

\section{PATIENT PERSPECTIVE ON nOH SYMPTOMS}

"Initially, I did not realize that I had nOH. I would have a tendency to be slow getting started in a movement, getting sleepy during the day and dozing, overall lethargic feeling, difficult to get motivated.... In the morning in getting dressed and things like that, I would be frozen, and during the day, I would get sleepy. I would try to get up and walk around, but it was hard to get up.... As the day progressed, I became slower and slower and the symptoms were more pronounced.... Sometimes, I would go to bed at 8:00 pm, which is abnormal for me. It was difficult to plan things for the evening after work... [It was] very hard to get motivated, and [I had] a desire to just go to bed.... Looking back on it, I think it had an impact on my cognitive behavior.... This [led] to depression, loss of sleep, anxiety, and overall malaise."

\section{ROLE OF HCPS IN nOH CARE}

Although HCPs treating patients at risk of $\mathrm{nOH}$ are well suited to recognize symptoms and facilitate discussions regarding $\mathrm{nOH}$ with patients and their caregivers (Fig. 1) [2, 3, 8, 9], they also need to understand potential communication challenges. Patients with $\mathrm{nOH}$ may hide or minimize their symptoms and not discuss them with their HCP unless the symptoms are severe [4]. Further, patients may attribute the symptoms to the underlying neurodegenerative disease or other comorbidities, not realizing that $\mathrm{nOH}$ is a separate condition. Others may be concerned that reporting $\mathrm{nOH}$ symptoms or falls could lead to restrictions on their activities and ability to live independently [2]. Patients may not always be forthcoming regarding $\mathrm{nOH}$ symptoms, and patient survey data have indicated that patients find the path to diagnosis challenging. Half of patients with $\mathrm{nOH}$ reported frustration with the diagnostic process, which may have included multiple HCP consultations before their eventual diagnosis (43\% consulted $\geq 3$ HCPs) [4]. Increased awareness of these issues will better enable HCPs to diagnose and treat these bothersome symptoms.

When $\mathrm{nOH}$ is suspected, orthostatic vital signs should be taken to observe whether the BP drop is indicative of $\mathrm{OH}(\geq 20 \mathrm{mmHg}$ systolic or $\geq 10 \mathrm{mmHg}$ diastolic) $[2,3]$. However, individual in-clinic evaluations of orthostatic vitals might not confirm $\mathrm{OH}$, and an at-home BP and symptom diary may be required [2]. This diary should include the time of each BP measurement and accompanying circumstances, such as recent meals, timing of medications, or exercise. In patients with $\mathrm{PD}$, such a record can also be used to document "on" and "off" periods of 


\section{Recognize Individuals at Risk of $\mathrm{nOH}$}

- Parkinson disease, multiple system atrophy, pure autonomic failure, or other neuropathies

- Older age ( $\geq 70 \mathrm{y}$ ), frail, or polypharmacy use

- Unexplained syncope or falls

- Symptoms that occur only when standing

\section{Ask About Orthostatic Symptoms}

-When standing, do you experience

- Falls?

- Leg buckling/weakness?

- Dizziness/lightheadedness?

- Confusion?

- Vision disturbances?

- Other symptoms/"not feeling right"?

- Neck pain/aching?

- How long can you stand and/or walk unassisted?

- Relationship and effect of symptoms on daily activities

- Symptoms exacerbated by meals, exercise, or during certain times (eg, morning, nocturnal visits to the bathroom)?

- Effect of symptoms on daily activities?

\section{Diagnose $\mathrm{OH}$}

- Identify OH (SBP drop of $\geq 20 \mathrm{mmHg}$ or DBP $\geq 10 \mathrm{mmHg}$ after $\geq 3$ min of standing or head-up tilt) by measuring orthostatic BP

- Consider an at-home BP and symptom diary

\section{Evaluate for Neurogenic Cause (Diagnose nOH)}

- Rule out nonneurogenic causes (eg, conduct physical examination, patient history, electrocardiogram, laboratory testing)

- An orthostatic HR increase $<15$ bpm is associated with $\mathrm{nOH}$

- Consider referral for specialized autonomic function testing

\section{Evaluate Medications Used}

Medications with potentially hypotensive effects

- Alpha blockers (eg, afuzosin, doxazosin prazosin, terazosin, tamsulosin)

- Calcium channel blockers (eg, amlodipine, nifedipine, diltiazem)

- Alpha-beta blockers (eg, carvedilol, labetalol)

- Angiotensin II receptor blockers (eg, losartan)

- Angiotensin converting enzyme inhibitors (eg, captopril, enalapril)

- Antidepressants (especially tricyclic agents like amitriptyline or nortriptyline)

- Central acting alpha-2 agonists (eg, clonidine)

- Diuretics (eg, furosemide, hydrochlorothiazide, spironolactone)

- Dopaminergic agents (eg, levodopa)

- Phosphodiesterase inhibitors (eg, sildenafil, tadalafil)

- Beta blockers (eg, metoprolol, atenolol) — - Vasodilators (eg, hydralazine, minoxidil)

Consider changing medication regimen to alleviate hypotension (eg, dose reduction, adjusting timing of use, discontinuation) in consultation with care team

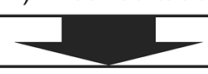

\section{Manage nOH Symptoms}

- Non-pharmacologic management

- Increased intake of salt and fluid

- Use of compression garments/abdominal binders

- Gentle exercise in recumbent position

- Sleep and rest in a head-up tilt position

- Pharmacologic management

- Droxidopa (FDA approved to treat the symptoms of $\mathrm{nOH}$ due to autonomic dysfunction)

- Midodrine (FDA approved to treat symptomatic OH) 
4 Fig. 1 Stepwise approach to care of patients with $\mathrm{nOH}$ $[2,3,8,9] . B P$ blood pressure, DBP diastolic blood pressure, FDA US Food and Drug Administration, $H R$ heart rate, $n \mathrm{OH}$ neurogenic orthostatic hypertension, $\mathrm{OH}$ orthostatic hypertension, SBP systolic blood pressure

dopaminergic medication, allowing the HCP a comprehensive view of the patient's overall symptom experience. Even after initial recognition of $\mathrm{OH} / \mathrm{nOH}$, continued use of a symptom diary can be useful to guide and adjust the treatment plan over time.

Once the presence of $\mathrm{OH}$ has been established, HCPs should aim to reduce any nonneurogenic causes of OH (i.e., Fig. 1, Step 4). For example, the potential contributing effects of any concomitant medications should be considered. Patients with PD and other synucleinopathies are often treated with medications such as levodopa (or other dopaminergic agents) and monoamine oxidase inhibitors that are associated with hypotensive effects and may worsen $\mathrm{nOH}$ symptoms [2, 10]. Additionally, many other medications commonly used in older populations also have hypotensive effects, including drugs for hypertension, depression, erectile dysfunction, benign prostatic hyperplasia (BPH; alfuzosin or tamsulosin), or hair loss (minoxidil) [2]. In consultation with the care team, use of these medications in patients with nOH should be modified if possible (e.g., dose reduction, adjustment of timing of use [particularly PD medications], discontinuation) without exacerbating the indicated conditions [2]. Referral for specialized autonomic function testing may be appropriate if the diagnosis continues to be unclear.

When managing nOH symptoms, attaining a certain standing blood pressure is not necessary; rather, the goals of treatment should focus on patient outcomes, including decreasing $\mathrm{nOH}$ symptom burden, mitigating risk of falls and fall-related injuries, and improving the ability to function independently in daily activities [2]. The precise treatment choices should be tailored to the individual patient's needs and circumstances considering both non-pharmacologic and pharmacologic options.
Non-pharmacologic measures are an important component of $\mathrm{nOH}$ management, but patients may not recognize or readily implement these measures. For example, waist-high compression garments are effective for treating patients with $\mathrm{nOH}$, but patients may be reluctant to wear them because they may find these compression garments uncomfortable (especially in warmer weather) [2]. Patients may also be inadequately hydrated from use of diuretics or self-restricted fluid intake to reduce urinary urgency or frequency related to stress incontinence, $\mathrm{BPH}$, or other bladder disorders $[2,11]$. In these cases, HCPs can educate patients to encourage compliance and enlist the help of the caregiver if necessary.

Although non-pharmacologic measures are often used as the initial approach to treat $\mathrm{nOH}$, implementing pharmacotherapy is often necessary for adequate relief of symptoms. Droxidopa and midodrine are approved by the US Food and Drug Administration to treat the symptoms of $\mathrm{nOH}$ and $\mathrm{OH}$, respectively (Fig. 1) $[8,9]$. Other agents (e.g., fludrocortisone, pyridostigmine) are commonly used off-label for the treatment of $\mathrm{nOH}[2,12,13]$ but have limited evidence of efficacy based on results from well-designed trials. Several comprehensive reviews on the management of $\mathrm{nOH}$ have been published in the past 3 years and are useful resources for detailed guidance and specific considerations of individual non-pharmacologic and pharmacologic interventions $[2,12,13]$.

\section{PATIENT PERSPECTIVE ON nOH CARE}

"I attributed all of [the symptoms] to PD. It became evident that the nOH was causing me to feel more 'stuck' or 'frozen' than I would otherwise feel as a Parkinson's patient. There is so much information that [my HCPs] had, and you just get overwhelmed after a bit and you don't necessarily connect the dots that [BP] may be an issue... as a non-medical person, I don't know what factors matter and what to tell [my HCPs]. Or I might get confused as to why something is happening. So I just am very 
careful not to put any judgment on what I'm feeling when I'm telling them my symptoms. [My HCPs] are very good at asking the same question several different ways to clarify the answers.

I would suggest... to keep a journal of BP, medication changes, environmental changes, and a detailed account of any changes in how [you] are feeling and/or functioning... an [HCP] might be able to look at it and say 'this guy could have low blood pressure.'

Once we were able to recognize that $\mathrm{nOH}$ was an issue [and treatment was initiated], within a few days, I began to perk up, had a bounce in my step, felt overall motivated to exercise and take on projects, slept better, and felt very encouraged. After taking the medication, I've been feeling more active and more resilient. You begin to have hope. The improvement in energy levels and the decline of depression and anxiety were great. I felt like my cognitive skills were returning to preParkinson's level."

\section{CAREGIVER PERSPECTIVE ON $\mathrm{nOH}$ CARE}

"I notice a huge difference... his attitude, the way he's getting up and down; he's been much better. I think he was beginning to think he couldn't do stuff. Once [his HCPs] figured it out, I think his attitude changed."

\section{CONCLUSIONS}

Patients with nOH may experience burdensome symptoms, functional impairments, and a decreased sense of psychosocial well-being. With increased awareness of the recognition, diagnosis, and management of nOH, HCPs can play a key role in improving clinical outcomes and quality of life in the patients they treat.

\section{ACKNOWLEDGEMENTS}

The authors thank the patient and his caregiver (wife) for their participation in this project.
Funding. Lundbeck (Deerfield, IL) provided funding for editorial support for manuscript preparation and the Rapid Service and Open Access fees.

Authorship. All named authors meet the International Committee of Medical Journal Editors criteria for authorship for this article, take responsibility for the integrity of the work as a whole, and have given their approval for this version to be published.

Medical Writing, Editorial, and Other Assistance. Medical writing and editorial assistance in the preparation of this article was provided by Lauren Stutzbach, PhD, and Lisa Havran, PhD, of ICON (North Wales, PA), which was supported by Lundbeck.

Disclosures. Jessie S. Gibson has served as a consultant for Lundbeck and received research support and compensation for speaker bureau participation from Teva Neuroscience. Christine B. Hunter has served on advisory boards for Acorda Therapeutics and Lundbeck. L. Arthur Hewitt is an employee of Lundbeck.

Compliance with Ethics Guidelines. Institutional review board approval was not required for this single-patient case study. Informed consent to share this case was obtained from the patient and his caregiver (wife). All patient information has been anonymized.

Prior Presentation. Aspects of this work were previously presented as a poster at the National Conference for Nurse Practitioners; September 24-27, 2019; Las Vegas, NV.

Data Availability. Data sharing is not applicable to this article as no datasets were generated or analyzed during the current study.

Open Access. This article is licensed under a Creative Commons Attribution-NonCommercial 4.0 International License, which permits any non-commercial use, sharing, adaptation, distribution and reproduction in any medium or format, as long as you give appropriate credit to the original author(s) and 
the source, provide a link to the Creative Commons licence, and indicate if changes were made. The images or other third party material in this article are included in the article's Creative Commons licence, unless indicated otherwise in a credit line to the material. If material is not included in the article's Creative Commons licence and your intended use is not permitted by statutory regulation or exceeds the permitted use, you will need to obtain permission directly from the copyright holder. To view a copy of this licence, visit http://creativecommons.org/ licenses/by-nc/4.0/.

\section{REFERENCES}

1. Savica R, Grossardt BR, Bower JH, Ahlskog JE, Rocca WA. Incidence and pathology of synucleinopathies and tauopathies related to parkinsonism. JAMA Neurol. 2013;70(7):859-66.

2. Gibbons $\mathrm{CH}$, Schmidt P, Biaggioni I, et al. The recommendations of a consensus panel for the screening, diagnosis, and treatment of neurogenic orthostatic hypotension and associated supine hypertension. J Neurol. 2017;264(8):1567-82.

3. Freeman R, Wieling W, Axelrod FB, et al. Consensus statement on the definition of orthostatic hypotension, neurally mediated syncope and the postural tachycardia syndrome. Clin Auton Res. 2011;21(2):69-72.

4. Claassen DO, Adler CH, Hewitt LA, Gibbons C. Characterization of the symptoms of neurogenic orthostatic hypotension and their impact from a survey of patients and caregivers. BMC Neurol. 2018;18(1):125.

5. Merola A, Sawyer RP, Artusi CA, et al. Orthostatic hypotension in Parkinson disease: impact on health care utilization. Parkinsonism Relat Disord. 2017;47:45-9.

6. Francois C, Biaggioni I, Shibao C, et al. Fall-related healthcare use and costs in neurogenic orthostatic hypotension with Parkinson's disease. J Med Econ. 2017;20(5):525-32.

7. LeWitt PA, Kymes S, Hauser RA. Parkinson disease and orthostatic hypotension in the elderly: recognition and management of risk factors for falls. Aging Dis. 2020;11(3):679-91.

8. Northera ${ }^{\circledR}$ (droxidopa). Full prescribing information. Deerfield: Lundbeck NA Ltd; 2017.

9. ProAmatine ${ }^{\circledR}$ (midodrine hydrochloride). Full prescribing information. Lexington: Shire US Inc.; 2017.

10. Cutsforth-Gregory JK, Low PA. Neurogenic orthostatic hypotension in Parkinson disease: a primer. Neurol Ther. 2019;8(2):307-24.

11. Weinberg AD, Minaker KL. Dehydration. Evaluation and management in older adults. Council on Scientific Affairs, American Medical Association. JAMA. 1995;274(19):1552-6.

12. Freeman $\mathrm{R}$, Abuzinadah $\mathrm{AR}$, Gibbons $\mathrm{C}$, Jones $\mathrm{P}$, Miglis MG, Sinn DI. Orthostatic hypotension: JACC state-of-the-art review. J Am Coll Cardiol. 2018;72(11):1294-309.

13. Olshansky B, Muldowney J. Cardiovascular safety considerations in the treatment of neurogenic orthostatic hypotension. Am J Cardiol. 2020;125(10):1582-93. 